\title{
First spectral measurements of a cryogenic high-field short-period undulator
}

\author{
F. Holy, ${ }^{1,2, *}$ A. R. Maier, ${ }^{1,2,3}$ B. Zeitler, ${ }^{1,3}$ R. Weingartner, ${ }^{2}$ S. Raith, ${ }^{2}$ N. Kajumba, ${ }^{2}$ \\ M. El Ghazaly, ${ }^{4,5}$ W. Lauth, ${ }^{4}$ D. Krambrich, ${ }^{4}$ A. Gaupp, ${ }^{6}$ M. Scheer, ${ }^{6}$ \\ J. Bahrdt, ${ }^{6}$ and F. Grüner ${ }^{1,2,3, \uparrow}$ \\ ${ }^{1}$ Institut für Experimentalphysik, Universität Hamburg, 22607 Hamburg, Germany \\ ${ }^{2}$ Department für Physik, Ludwig-Maximilians Universität, 85748 Garching, Germany \\ ${ }^{3}$ Center for Free-Electron Laser Science, Notkestrasse 85, 22607 Hamburg, Germany \\ ${ }^{4}$ Institut für Kernphysik, Johannes Gutenberg-Universität Mainz, 55128 Mainz, Germany \\ ${ }^{5}$ Department of Physics, Faculty of Science, Zagazig University, 44519 Zagazig, Egypt \\ ${ }^{6}$ Helmholtz-Zentrum Berlin für Materialien und Energie, 14109 Berlin, Germany
}

(Received 18 June 2013; revised manuscript received 3 April 2014; published 27 May 2014)

\begin{abstract}
Further development of synchrotron light sources and new concepts for free electron lasers require undulators with short periods and high magnetic fields. A promising approach is the cryogenic permanent magnet undulator concept based on an advanced magnet material. This new rare earth alloy $(\mathrm{Pr}, \mathrm{Nd})_{2} \mathrm{Fe}_{14} B$, shows an increasing remanent field of up to $1.7 \mathrm{~T}$ without the limits of spin reorientation transition. This work presents first spectral measurements of a prototype cryogenic permanent magnet undulator, consisting of 20 periods of $9 \mathrm{~mm}$ in length, cooled by a closed cycle cryo-cooler to temperatures below $30 \mathrm{~K}$. The $K$ parameter of 0.837 at RT is increased by more than $15 \%$ to 0.966 , and an increase of the third harmonics photon flux of up to $66 \%$ was achieved. A possible degradation of the on-axis field quality due to thermally induced magnetic field errors, deduced from the measured bandwidth of the spectrum, is below the limits of the detector resolution of $2 \%$.
\end{abstract}

\section{INTRODUCTION}

An advanced understanding of Nature's fundamental structure and processes requires electromagnetic radiation with a short wavelength and pulse duration. The most common sources for brilliant and quasimonochromatic light in the soft and hard X-ray regime are undulators used as insertion devices in electron storage rings or as single pass devices at linear accelerators. Recent case studies $[1,2]$ have shown that undulators with short periods offer advantages for different kinds of synchrotron light sources and free electron laser (FEL) schemes when they provide sufficiently high magnetic fields. Using these highfield short-period undulators, existing synchrotron light source facilities can extend their achievable photon energy range to the hard X-ray regime. Further, these new undulator designs are essential for the approach to future concepts of X-ray free electron lasers (XFELs) using laser based electron acceleration [3]. A short undulator period enables short photon wavelengths at moderate electron

\footnotetext{
*florian.holy@physik.uni-muenchen.de

†florian.gruener@desy.de
}

Published by the American Physical Society under the terms of the Creative Commons Attribution 3.0 License. Further distribution of this work must maintain attribution to the author $(s)$ and the published article's title, journal citation, and DOI. energies and a short FEL gain length while a high field increases the acceptance of electron energy spread.

To improve upon current state-of-the-art short period undulators, two options are in discussion: First, the use of superconducting (SC) materials (e.g., SC wire solenoids) and second the use of rare-earth (RE) permanent magnets cooled to temperatures around $140 \mathrm{~K}$. The SC concept promises outstanding magnetic properties beyond the theoretical limits of RE permanent magnet materials around $1.8 \mathrm{~T}$ for the remanence. But even state-of-the-art $\mathrm{SC}$-materials $\left(\mathrm{NbTi}, \mathrm{Nb}_{3} \mathrm{Sn}\right)$ require temperatures around $4 \mathrm{~K}$ to enable critical current densities above $1000 \mathrm{~A} / \mathrm{mm}^{2}$. Associated with this are high costs for the cooling infrastructure due to a high heat load at temperatures where commercially available cryo-coolers are inefficient and expensive. Another drawback is the lack of a field optimizing shimming concept which still has to be developed. Recently J. Bahrdt [4] gave a thorough comparison of both technologies utilizing state-of-the-art materials. The second option is the use of permanent magnets based on RE elements like Neodymium (Nd), Dysprosium (Dy), and Praseodymium (Pr). These magnets achieve an increase of remanence and coercivity when they are cooled down. But commonly used RE magnets, Nd and Dy based materials, reach a maximum gain in their remanence of about $8 \%$ at temperatures around $140 \mathrm{~K}$ and lose flux density below that temperature due to a spin reorientation transition [5-7]. 
Recently, Uestüner et al. [5,8] proposed an optimized composition of RE materials with an $80 \%$ ratio of $\mathrm{Pr}$ in the RE-part. This magnet material avoids spin reorientation and shows a monotonic gain of more than $16 \%$ in remanence, reaching 1.69 T. This field strength, close to the crystal's theoretical limit, is achieved at temperatures around $30 \mathrm{~K}$. The coercivity of the new material increases by a factor of three to about $5700 \mathrm{kA} / \mathrm{m}$ at cryogenic temperatures. Since the coercivity is a measure for the magnets resistivity against demagnetization by irradiation, the cryogenic permanent magnet undulator (CPMU) is expected to provide a significantly increased lifetime, compared to standard permanent magnet undulators, thus reducing costs and maintenance work, offering higher reliability $[9,10]$.

A collaboration of Helmholtz-Zentrum Berlin (HZB) and the experimental physics department of the University of Hamburg (UHH) initiated the development of an undulator with this novel permanent magnet material. The undulator was designed and assembled at the HZB. This work presents the first operation of a prototype CPMU built with the new material composition $\left(\mathrm{Nd}_{0.2}, \mathrm{Pr}_{0.8}\right)_{2} \mathrm{Fe}_{14} B$. The spectral measurements were done during a beam time at Mainzer Mikrotron (MAMI). The following Sec. II introduces to the main characteristics of undulator radiation (UR), followed by Sec. III, in which the magnetic field characteristics of the prototype undulator are presented, measured by a newly developed cryogenic Hall-probe bench at the HZB [11]. Section IV presents the experimental setup. Two independent detectors observed the UR with respect to the discussed characteristics. The results of the spectral measurements are compared to the magnetic field measurements in Sec. V. Section VI sums up the results and gives an outlook to future projects.

\section{UNDULATOR RADIATION CHARACTERISTICS}

Both wavelength tunability as well as photon flux or rather brilliance-defined as number of photons divided by their phase space volume-specify the applicability and versatility of scientific light sources. Both wavelength and photon yield generated by undulators depend on the undulator's period length and the magnetic field. These properties are combined to the undulator parameter $K=$ $e \tilde{B} \lambda_{u} /\left(2 \pi m_{e} c\right) \approx 0.9336 \tilde{B}[T] \lambda_{u}[\mathrm{~cm}]$ as a characteristic measure for undulators [12]. Here $e$ is the elementary charge, $\tilde{B}$ the magnetic peak field on-axis, $\lambda_{u}$ the undulator's period length, and $m_{e}$ the electron's rest mass.

To characterize the newly developed CPMU prototype, the following spectral characteristics of the UR were measured, in order to determine the undulator $K$ parameter, the error of $K$ and its gain when cooled down to cryogenic temperatures.

\section{A. Peak wavelength}

The wavelengths of the UR harmonics are determined by the undulator equation [13]:

$$
\lambda_{\mathrm{ph}}=\frac{\lambda_{u}}{2 n_{u} \gamma^{2}}\left(1+\frac{K^{2}}{2}+\theta^{2} \gamma^{2}\right) .
$$

Here $\lambda_{\text {ph }}$ is the emitted photon wavelength, $\lambda_{u}$ the period length of the undulator, $K$ the characteristic undulator parameter and $\gamma=1+E /\left(m_{0} c^{2}\right)$ the Lorentz factor describing the total energy of the electrons in the beam. $n_{u}=1,2,3, \ldots$ is the harmonic number.

For odd harmonic numbers $n_{u}$ the photons are emitted in a cone of light around the electrons direction of motion, whereas the even harmonics do not appear on-axis. The spatial distribution of the odd harmonics can be approximated by a Gaussian distribution with an opening angle of $\sigma_{r^{\prime}}=\sqrt{\lambda_{\mathrm{ph}} / 2 L_{u}}$ [13]. Here $L_{u}$ is the total length of the undulator. The last term of Eq. (1) describes the redshift due to the relativistic Doppler effect at observation angles $\theta$. This effect also causes a spectrometer with a finite aperture to detect a certain amount of radiation with red shifted peak wavelengths. If the UR is integrated over the whole radiation cone, this shift is described by:

$$
\lambda_{\text {peak }}=\frac{\lambda_{1}}{n_{u}\left(1-1 / n_{u} N_{u}\right)},
$$

where $N_{u}$ is the number of undulator periods and $\lambda_{1}$ the fundamental wavelength of the radiation spectrum [14].

\section{B. Bandwidth}

From the interference condition of the UR, emitted at every period over the whole undulator length, one obtains the intrinsic bandwidth of each harmonic peak [13]:

$$
\left(\frac{\Delta \lambda}{\lambda}\right)_{U}=\frac{1}{n_{u} N_{u}} \quad\left(\text { for } n_{u}=1,3,5, \ldots\right) .
$$

Additionally one gets a contribution from magnetic field errors $\Delta B$. From Eq. (1) follows:

$$
\left(\frac{\Delta \lambda}{\lambda}\right)_{B}=\frac{K^{2}}{\left(1+\frac{K^{2}}{2}\right)} \frac{\Delta B}{B}, \quad \text { with } \quad \frac{\Delta B}{B}=\frac{\Delta K}{K} .
$$

Since these bandwidth broadening effects are statistically independent, the total bandwidth of UR on-axis is given by a quadratic sum, Eqs. (3) and (4).

\section{Photon flux density}

The on-axis photon flux density in units of photons per solid angle, second and $0.1 \%$ bandwidth for an electron beam with zero divergence can be approximated [13] by: 


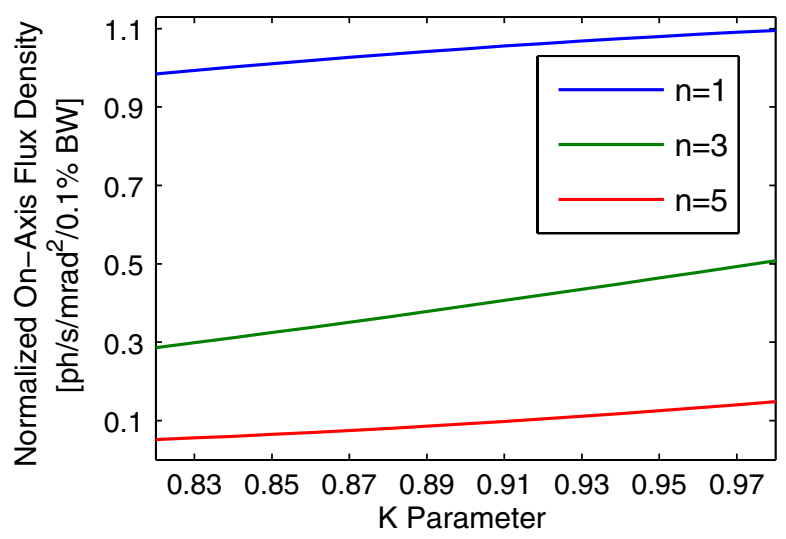

FIG. 1. On-axis photon flux density of UR, according to Eq. (5), normalized to the measured $K$ parameter at RT. Theory predicts a relative gain in photon flux density of $9 \%, 66 \%$, and $100 \%$ for the $1 \mathrm{st}, 3 \mathrm{rd}$, and 5 th harmonic, respectively, if $K$ is increased from 0.837 at RT to 0.966 at $29 \mathrm{~K}$.

$$
\left.\frac{d \dot{N}}{d \Omega}\right|_{\theta=0} \approx 1.74 \times 10^{14} N_{u}^{2} E^{2} I F_{n}(K) .
$$

Here $\dot{N}$ is the number of photons per second, $E$ the electron energy, $I$ the average electron beam current, and $F_{n}(K)$ a sum of modified Bessel functions $J . F_{n}(K)$ is defined by $n_{u}^{2} K^{2} /\left(1+K^{2} / 2\right)^{2} \times\left[J_{\left(n_{u}+1\right) / 2}(Y)-\right.$ $\left.J_{\left(n_{u}-1\right) / 2}(Y)\right]^{2}$ with $Y=n_{u} K^{2} /\left[4\left(1+K^{2} / 2\right)\right]$. Figure 1 shows the increase of the on-axis flux density for a $K$ parameter increase from 0.82 to 0.98 generated by a zero divergence electron beam, according to Eq. (5). The plot is normalized to the value of the fundamental of $K=0.83$. However, it describes only UR generated by zero divergence electron beams. For divergent electron beams a modification of Eq. (5) must be made, as suggested by P. Elleaume [13]:

$\left.\left.\frac{d \dot{N}\left(\sigma_{x^{\prime}}, \sigma_{z^{\prime}}\right)}{d \Omega}\right|_{\theta=0} \approx \frac{d \dot{N}}{d \Omega}\right|_{\theta=0}\left[\left(1+\frac{L \sigma_{x^{\prime}}^{2}}{2 \lambda_{\mathrm{ph}}}\right)\left(1+\frac{L \sigma_{z^{\prime}}^{2}}{2 \lambda_{\mathrm{ph}}}\right)\right]^{-1}$.

Here $\sigma_{x^{\prime}}$ and $\sigma_{z^{\prime}}$ are the horizontal and vertical electron divergences, $L$ the undulator length, and $\lambda_{\text {ph }}$ the photon wavelength.

\section{UNDULATOR SPECIFICATIONS}

The new magnet material as proposed by $[5,6]$ is based on an alloy of $\mathrm{Pr}$ and $\mathrm{Nd}$ and optimized for use in an undulator. The coercivity was chosen to be sufficiently high to guarantee a stable magnetization in the assembled structure at RT. During assembly the environmental temperature was chosen to be $-20^{\circ} \mathrm{C}$ (cold house assembly) to avoid demagnetization by opposing fields. These fields are highest for a magnet which is moved from outside to inside the girder. Later, it has been demonstrated that the new
TABLE I. Specifications of the CPMU prototype undulator. The magnetic peak field $\tilde{B}$ is measured at HZB with a Hall-probe bench, the magnet's remanence $B_{r}$ is deduced from measurements using Helmholtz coils.

\begin{tabular}{lcc}
\hline \hline Specifications & $300 \mathrm{~K}$ & $25 \mathrm{~K}$ \\
\hline$B_{r}$ & $1.42 \mathrm{~T}$ & $1.69 \mathrm{~T}$ \\
$\tilde{B}_{K}$ & $0.996 \mathrm{~T}$ & $1.149 \mathrm{~T}$ \\
$K$ & 0.837 & 0.966 \\
$\lambda_{u}$ & \multicolumn{3}{c}{$9 \mathrm{~mm}$} & \\
$N$ & \multicolumn{3}{c}{20} \\
Gap & $2.5 \mathrm{~mm}$ \\
\hline \hline
\end{tabular}

material can be treated with a grain boundary diffusion process which enhances the coercivity and permits assembly at RT [15].

The support structure of the prototype is made of oxygen free high conductivity copper (OFHC) to maximize the thermal conductivity. As the temperature lowers, heat transfer by phonon scattering becomes less efficient. But for highly purified OFHC copper, the electric conductivity at cryogenic temperatures increases by three orders of magnitude. At these temperatures, electrons from the conduction band are no longer disturbed by the oscillations of the metal's crystal lattice and the majority of heat is now transported by these free moving electrons. Thus the low electric resistance of the support structure at low temperatures helps avoid thermal gradients along the undulator and enables a fast cooling process.

Magnetization measurements of each undulator magnet taken before assembly and a subsequent matching of neighbor magnets in the undulator together with sub micrometer processing accuracy, lead to a relative rms field error of the assembled device below $1.75 \%$ corresponding to a phase error below $3.7^{\circ}$ both measured at $15 \mathrm{~K}$ [16]. The specifications measured by an in vacuum Hallprobe bench are listed in Table I.

\section{SETUP}

In order to investigate the characteristics of the undulator prototype, first measurements of its spectral output were performed at a conventional electron accelerator. Using the 855.1 MeV beam line of MAMI-B, the flux, wavelength, and line widths of the harmonic peaks at cryogenic and RTs were observed and compared. Two independent detector systems were used. One was a cryogenically cooled low energy resolving Germanium diode detector, the other was a gold wire transmission grating spectrometer (TGS). A schematic representation of the setup is given in Fig. 2.

The electron beam parameters were measured for both detector setups separately. A cross hair in combination with an ionization counter was used to determine the electron beam cross section at the undulator position by a so-called wire scan. It was later revealed that the resulting divergence applied only for the transmission grating measurements, 

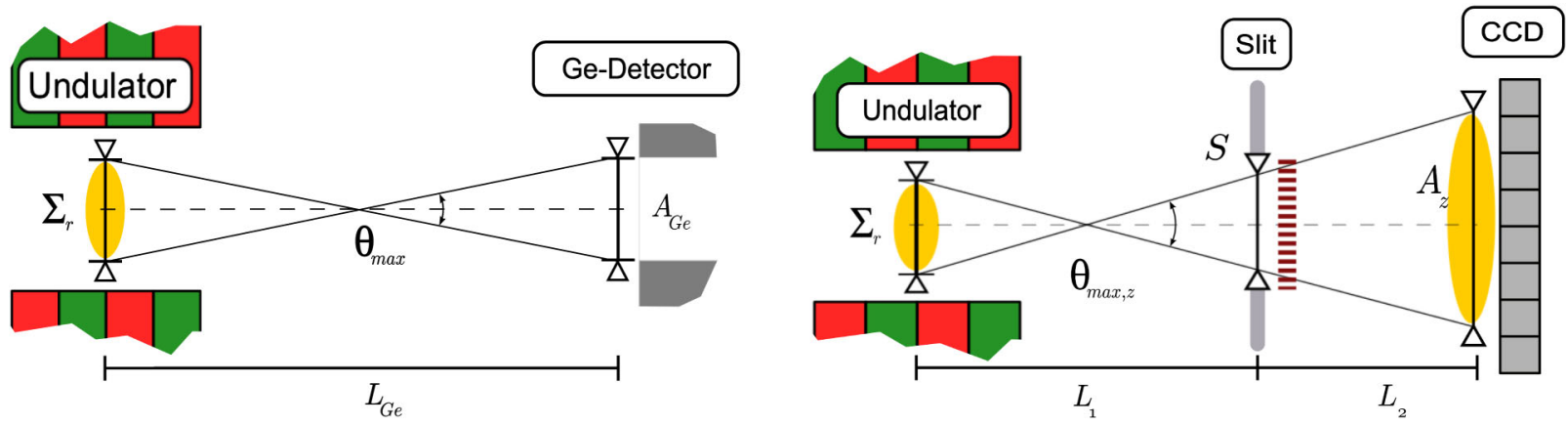

FIG. 2. Experimental setup: Two independent detector systems were used, a Ge-detector (left) and a transmission grating spectrometer (right). In both figures the radiation source size is represented by $\Sigma_{r}$, whereas $A$ is the detector aperture and $\theta_{\text {max }}$ the resulting acceptance angle. The transmission grating spectrometer on the right-hand side has an entrance slit of size $S$ which restricts the angle of incidence $\theta_{\max , z}$ to optimize the spectral resolution. The distances are represented by $L$.

which were performed immediately before the wire scan. The divergence during the Ge-detector measurements was determined by an analysis of transverse scans of the UR cones. These scans were performed with the Ge-detector itself shortly before the spectra presented in Fig. 3 were measured. Observing systematically the radiation intensity at the theoretical resonance energy of the fundamental for several off-axis detector positions, showed that the divergence during the Ge-detector measurements was significantly higher than the results of the wire scan. The electron beam parameters for both setups are summed up in Tables II and III calculated assuming a normalized beam emittance of $(0.5 / 7.8) \pi \mathrm{nm} \mathrm{rad}$ (vertical/horizontal) at $855.1 \mathrm{MeV}$ energy [17].

The undulator was cooled by a single-stage GiffordMcMahon (GM) cycle cryo-refrigerator. With a simple heat radiation shield made of Super insulation the undulator was cooled down to a temperature of $25 \mathrm{~K}$. During 3 days of beam time the base temperature rose to $29 \mathrm{~K}$, probably caused by an increased attenuation coefficient, due to a

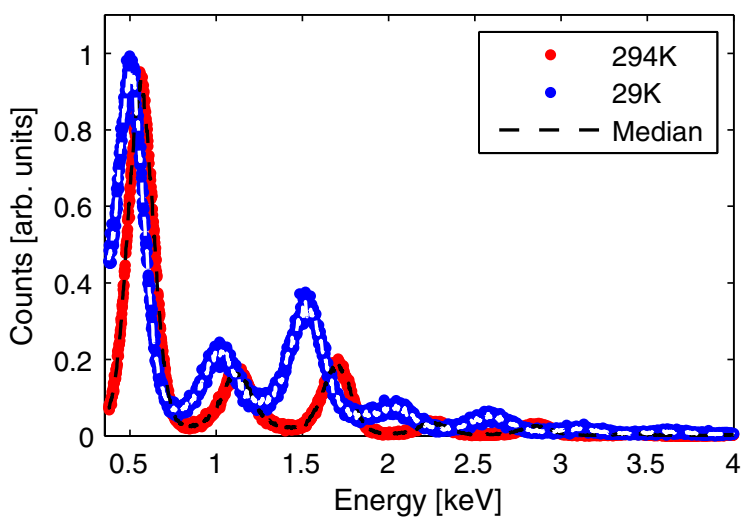

FIG. 3. Comparison of the undulator's spectral output at RT and at $29 \mathrm{~K}$ measured by a Germanium detector. The spectra are normalized to the peak count of the fundamental's flux density at RT. A shift of the peak energies and a gain in flux density due to the increasing $K$ parameter is observed. The measurement is in good agreement with Eq. (5). coating of out-frozen residual gas (vacuum pressure around $10^{-4} \mathrm{~Pa}$ ) at the surface of the undulator. The alignment of the undulator was done with two zinc sulfide screens attached to the undulator support and a camera.

\section{A. Germanium detector}

The Ge-detector was placed at $L_{\mathrm{Ge}}=13.4 \mathrm{~m}$ behind the undulator, equipped with a pinhole of $A_{\mathrm{Ge}}=500 \mu \mathrm{m}$ diameter. The pinhole and radiation source size $\Sigma_{r}$ define the angular acceptance of the detector: $\theta_{\max } \approx 39 \mu \mathrm{rad}$. Thereby a spatially resolved detection of the radiation was possible (radiation divergence $\approx 300 \mu \mathrm{rad}$ ). A transverse scan with the detector determined the divergence of the UR, as described in the last section. Thus it was possible to calculate the electron beam divergence deconvolving the intrinsic radiation divergence $\sigma_{r}$, described in Sec. II A. The result is listed in Table II. The energy resolution for a single photon $\Delta_{\mathrm{Ge}}$ is about $150 \mathrm{eV}$ (FWHM) [18], smaller than the spectral separation between two harmonic peaks $\left(\Delta_{E_{n}} \approx 500 \mathrm{eV}\right)$. Hence the harmonic structure of the spectrum is resolved. However the bandwidth of the spectral lines is about 5 times smaller than $\Delta_{\mathrm{Ge}}$ and thus not detectable. The calibration was done using decay spectra emitted by two different radioactive probes, $\mathrm{Fe}_{26}^{55}$ and $\mathrm{Am}_{95}^{243}$. To minimize the detector dead time and the number of multiphoton events, the electron beam current, and thereby the photon flux, was limited to maximum $10 \mathrm{pA}$.

TABLE II. Electron beam parameters during the Ge-detector measurements deduced from the size of the radiation cone.

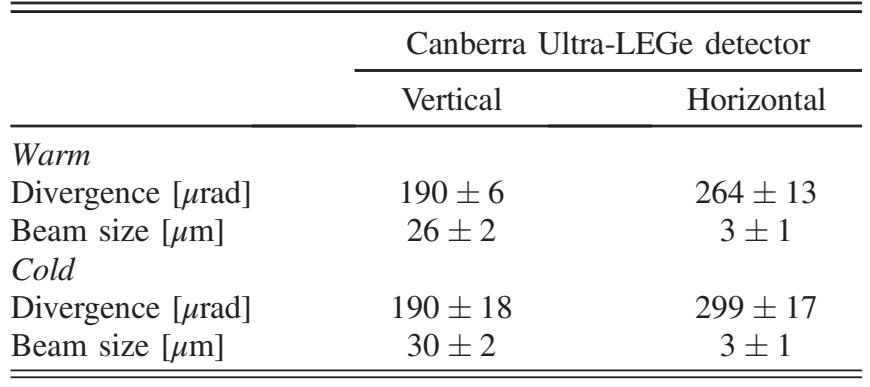


TABLE III. Electron beam parameters during the TGS measurements deduced from a wire scan at the undulator position.

\begin{tabular}{lcc}
\hline \hline \multicolumn{3}{c}{ Transmission grating spectrometer (TGS) } \\
\hline Warm \& cold & Vertical & Horizontal \\
\hline Divergence $[\mu \mathrm{rad}]$ & $3 \pm 1$ & $21 \pm 1$ \\
Beam size $[\mu \mathrm{m}]$ & $160 \pm 3$ & $367 \pm 7$ \\
\hline \hline
\end{tabular}

\section{B. Transmission grating}

The transmission grating spectrometer was based on a gold wire grating (1000 lines/mm [19]). An entrance slit with variable size, attached directly in front, restricted the angle of incidence toward the dispersion axis. The grating and the slit were placed $7.55 \mathrm{~m}\left(L_{1}\right)$ behind the undulator's center, leaving a dispersion drift of $5.85 \mathrm{~m}\left(L_{2}\right)$ to the X-ray CCD, as can be seen in Fig. 2.

Due to the pixel size of the CCD $\left(S_{\text {pix }}=22.5 \mu \mathrm{m}[20]\right)$ and positioning uncertainties the absolute wavelength accuracy is less than $2.5 \%$ at a wavelength of $0.4 \mathrm{~nm}$ (corresponding to the 5th UR harmonic) and $1 \%$ at $2 \mathrm{~nm}$ wavelength (corresponding to the UR fundamental). The spectral resolution is largely dominated by the projection of the extended source producing a spatially broad signal on the CCD. This results from the simplicity of the used setup without imaging features. An estimation of the spectral resolution of the transmission grating spectrometer has been made, considering geometric optics, Rayleigh's criterion and diffraction. With this resolution an increase of the magnetic field error due to thermally induced mechanical distortions should be detected if the additional field error (rms) becomes greater than 2\%. A more detailed description can be read in the Appendix.

\section{EXPERIMENTAL RESULTS}

The following measurements were done at RT, $294 \mathrm{~K}$ (red), and below $30 \mathrm{~K}$ (blue) in order to compare the performance of the new magnet material and to detect possible magnetic field errors generated by mechanical distortions due to the temperature drop.

First the Ge-Detector measurements are presented. Figure 3 shows the comparison of the measured on-axis photon flux densities, averaged over the detector's entrance window, at both temperatures. The curves are normalized to the flux density of the fundamental peak at RT. One can see a significant gain in the photon flux density due to the increased $K$-parameter. The measured gain of $9 \%, 70 \%$, and $100 \%$ for the fundamental, third, and fifth harmonics, respectively meets the expected gain for an increase of $K$ from 0.83 to 0.97 , given by Eq. (6). With the divergence determined by the transverse scans, shown in Table II, the theory predicts a resulting gain of $(1 \pm 24) \%,(46 \pm 33) \%$, and $(114 \pm 47) \%$ accordingly.

However, the theoretical values apply only directly on-axis (whereas the measurement averages over the detector's entrance window with an acceptance angle of $\left.\theta_{\max } \approx 39 \mu \mathrm{rad}\right)$. Furthermore, the flux density is highly sensitive to the roughly determined electron divergence. Thus a comparison between theory and measured values is only qualitatively possible. With this in mind one can state that the measured cold and warm UR spectral flux densities (Fig. 3) agree with the theory, and the values lay within the measurement's accuracy region.

Also the wavelengths of the peaks are analyzed to deduce the $K$ parameter at the given temperatures-see Fig. 4. Due to the finite aperture of the Germanium detector $\theta_{\max }$ and the redshift connected with it, the measurements were corrected by a detuning factor, given by Eq. (2). With this factor the measured values agree well with the undulator field measurements, done with a Hall-probe at the Helmholtz Zentrum Berlin, see Fig. 4.

The spectral bandwidths of the UR is determined by analyzing the CCD data of the transmission grating spectrometer-Fig. 5. Lineouts are shown in Fig. 6. The bandwidths are compared for warm and cold measurements and agree with the expected values, listed in Table IV, where broader bandwidths are predicted for the cold spectrum due to the longer wavelength of the harmonic peaks and the correspondingly lower spectrometer resolution. A potential broadening of the bandwidth of the fundamental, due to an additional field error, produced by mechanical stress and distortions, could not be detected. Neither was a temperature gradient along the undulator's magnets, generating a field error due to the magnet's temperature dependent remanence, detectable. Hence it is confirmed that an additional rms field error generated by these effects must be less than $2 \%$, given by the resolution limits of the used spectrometer. A more detailed description is given in the Appendix.

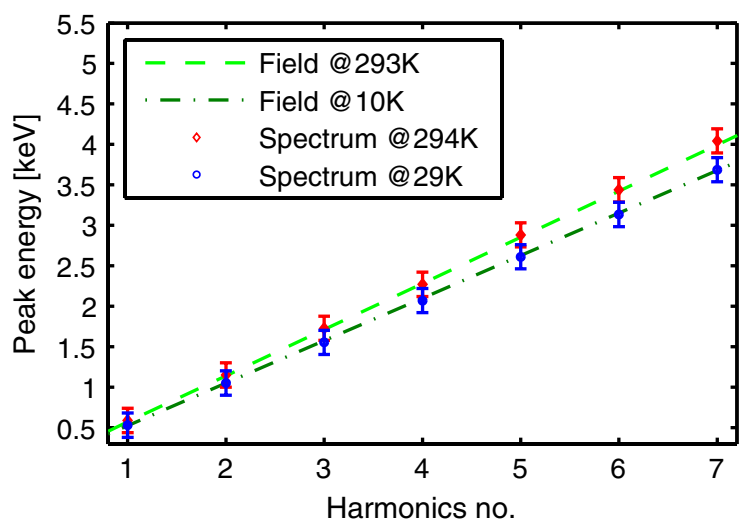

FIG. 4. The peak energies of the undulator spectrum measurement plotted against the corresponding harmonic number $n$. The data is detuning corrected, see Eq. (2), to compensate the redshift caused by the detector aperture. The dashed lines show the expected peak energies resulting from a magnetic field Hallprobe measurement done by the Helmholtz Zentrum Berlin. The error bars are given by the energy resolution of the Germanium detector of $150 \mathrm{eV}$ (FWHM). 

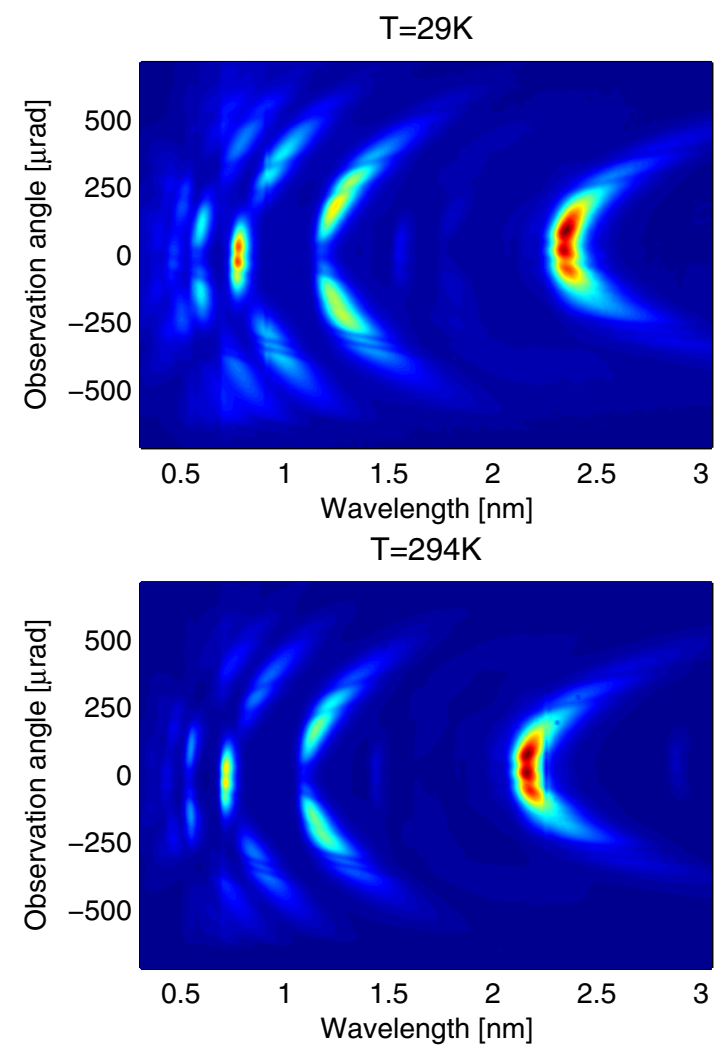

FIG. 5. CCD data from the TGS. The images compare the cooled case with the RT case. The entrance slit sizes $75 \mu \mathrm{m}$ and $100 \mu \mathrm{m}$ were adapted to optimize the resolution of the spectrometer for the specific wavelength of the first harmonic-see the Appendix. The color scale is normalized to the maximum count number of the fundamental peak. Shadows of the grating's support structure [19] are visible.

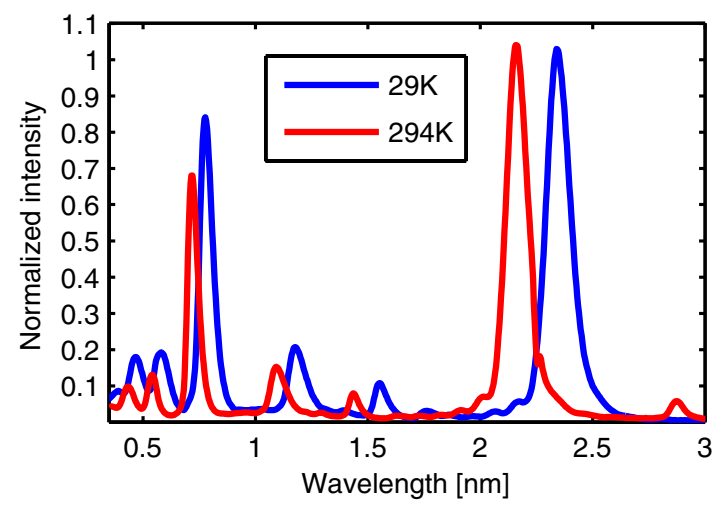

FIG. 6. Lineouts made at the center of the CCD images (observation angle $=0 \mathrm{rad}$, Fig. 5) of the cold (blue) and the warm (red) case. The absolute flux is not comparable since the entrance slits of the spectrometer are not the same in both cases. From these lineouts the bandwidth of the harmonics are determined and compared to the expected values, to examine a possible increase of field errors due to the temperature drop. The resulting bandwidths are given in Table IV. Both spectra show small peaks at two-thirds of the fundamental wavelength. These features are the third undulator harmonic diffracted into the gratings second order.
TABLE IV. Bandwidth of the UR measured with the transmission grating spectrometer. The fundamental is measured with a spectrometer's slit widths of $75 \mu \mathrm{m}$ and $100 \mu \mathrm{m}$ (warm and cold, respectively), the higher order bandwidths with $50 \mu \mathrm{m}$ slit widths for both temperatures. The bandwidth of the higher harmonics is overestimated by the theory (diffraction)-see appendix for details.

\begin{tabular}{lccccc}
\hline \hline \multirow{2}{*}{$\begin{array}{l}\text { Harmonic } \\
\text { No. }\end{array}$} & \multicolumn{2}{c}{ Warm $(293 \mathrm{~K})$} & & \multicolumn{2}{c}{ Cold $(25 \mathrm{~K})$} \\
\cline { 2 - 3 } \cline { 5 - 6 } & $\begin{array}{c}\text { Theory } \\
(\%)\end{array}$ & $\begin{array}{c}\text { Measured } \\
(\%)\end{array}$ & & $\begin{array}{c}\text { Theory } \\
(\%)\end{array}$ & $\begin{array}{c}\text { Measured } \\
(\%)\end{array}$ \\
\hline 1 & 5.2 & 5.2 & & 5.4 & 5.4 \\
3 & 13 & 7.6 & & 14 & 7.8 \\
5 & 22 & 17.3 & & 24 & 19.7 \\
\hline \hline
\end{tabular}

\section{CONCLUSION AND OUTLOOK}

The spectral measurements confirm the expected gain of the $K$ parameter of more than $15 \%$. Any additional source of field errors generated by mechanical distortion due to the temperature drop of more than $260 \mathrm{~K}$ or even produced by a thermal gradient along the undulator are below $2 \%$, corresponding to the resolution limit of our spectrometer. A significant gain of the photon flux was achieved and is in good agreement with the theory-9\%,70\%, and over $100 \%$ for the fundamental, third, and fifth harmonic's flux, respectively. The angle integrated photon flux increases up to $20 \%$ for all harmonics.

The gain of remanence leads to several possibilities for different UR sources. Existing third generation synchrotrons rely on the development of short-period undulators with high magnetic fields to increase their achievable photon energy range [21-24]. Furthermore, the use of the new permanent magnet material paves the way for the application of laser-wakefield accelerated electrons as source for UR and even as drivers for FELs. Here the 3 times increased coercivity of the cooled magnets and a correspondingly increased resistivity against demagnetization by irradiation with electrons and laser light is important since these newly developed laser-wakefield accelerators suffer from electron beam instabilities and a broad energy spread. Essential for the LWFA-FEL scenario is an increased $K$ parameter in combination with a relatively short period since thereby the interaction between electrons and light field is amplified and the acceptance of the electron's energy spread is increased [1].

Until now the described CPMU prototype was successfully used during two different beam times at MAMI and SLAC [25]. The next step will be the implementation into a laser-wakefield driven electron beam line. Due to the small undulator gap of $2.5 \mathrm{~mm}$ high but feasible standards in stability and control of the electron accelerator technology are required. Once this task is achieved, a longer insertion device with a shorter period length will follow, with the objective to establish a new kind of ultrabrilliant and tunable X-ray source for scientific purposes. 


\section{ACKNOWLEDGMENTS}

The author would like to thank the team that prepared and accomplished the beam time at MAMI, among them scientists and machine operators from UHH, LMU, MAMI, and HZB. Many thanks also for the support given by the workshops of MPQ and MAMI, who manufactured major parts of the experimental equipment. The Cluster of Excellence of the German Research Foundation, MunichCentre for Advanced Photonics (MAP), supported the beamtime financially and provided infrastructure. The cryogenic undulator project is funded by the BMBF Project No. 05K10WM2 and No. 05K10CB1.

\section{APPENDIX: SPECTRAL RESOLUTION OF THE TRANSMISSION GRATING SPECTROMETER}

The spectral resolution of the TGS is dominated by the size of the image of the spectrometer's entrance slit, projected onto the CCD—see Fig. 2. This size depends on the maximum divergence of the incident light along the dispersion axis. The maximum angle is given by: $\theta_{\max , z}=$ $\left(\Sigma_{r, z}+S\right) / L_{1}$ with the slit size $S$ and the standard deviation of the source size $\Sigma_{r, z}$. The spectral resolution depends therefore among other parameters on the size of the entrance slit $S$. If the size of the entrance slit $S$ is wide, the value of $A_{z}$, the projection of the source $\Sigma_{r}, z$ through the slit onto the CCD, becomes wide and overlapping of two nearby signals limits the resolution. On the other hand, Rayleigh's criterion limits the resolution for small entrance slits, since the number of illuminated grating grooves depends on $S$. Further, for small entrance slits $S$ and long wavelengths of the incident photons one has to take diffraction into account. For our case a Fresnel number $F$ around 1 indicates that near field effects and with it Fresnel diffraction dominates. The Fresnel number is given by $F=S^{2} /\left(4 \lambda_{\text {ph }} L_{2}\right)$. Now a simple approximation of the single slit diffraction can be made:

$$
\left(\frac{d \lambda_{\mathrm{ph}}}{\lambda_{\mathrm{ph}}}\right)_{\mathrm{tot}} \approx \sqrt{\left[\frac{d}{\lambda_{\mathrm{ph}}}\left(\frac{S}{L 2}+\frac{S+\Sigma_{r, x}}{L 1}\right)\right]^{2}+\left(\frac{1}{n_{g} N_{g}}\right)^{2}+\left(\frac{1}{n_{u} N_{u}}\right)^{2}+\left(2.78 \frac{d}{\pi S}\right)^{2}} .
$$

All mentioned effects are summarized by the following Gaussian sum which approximates the bandwidth measured by the TGS. The first summand of the Gaussian sum originates from geometric optics [26] as described above. The second term represents Rayleigh's criterion with the diffraction order $n_{g}$ and the number of illuminated slits $N_{g}$, while the third summand describes the intrinsic undulator bandwidth with harmonic number $n_{u}$ and the number of undulator periods $N_{u}$. The last term estimates the contribution due to single slit Fresnel diffraction. Two other contributions are neglected. The electrons' energy spread, below $0.2 \%$, is neglected as well as the contribution due to UR redshift. The redshift is limited by the acceptance angle of the spectrometer, which is set much smaller than the UR opening angle. The spectrometers' slit limits the acceptance angle $\theta_{\max , z}$ in the $z$ direction (see Fig. 2). The acceptance angle in the $x$ direction is given by the size of a single pixel and the distance between undulator and CCD. This is due to the fact that the bandwidth is determined by a lineout of the CCD data with a single pixel width. Therefore the acceptance angle in $x$ is given by $\theta_{\text {max }, x}=$ $\left(S_{\text {pix }}+\Sigma_{r, x}\right) /\left(L_{1}+L_{2}\right)$. These two angles, $\theta_{\text {max }, z} \approx$ $44 \mu \mathrm{rad}$ for a $100 \mu \mathrm{rad}$ slit and $\theta_{\max , x} \approx 30 \mu \mathrm{rad}$ for a source size $\Sigma_{r, x} \approx 370 \mu \mathrm{m}$, determine an additional contribution to the measured line width due to a certain amount of tilted radiation, which is also redshifted - see Eq. (1). The resulting contribution to the bandwidth is far below $0.5 \%$, and therefore also neglected.
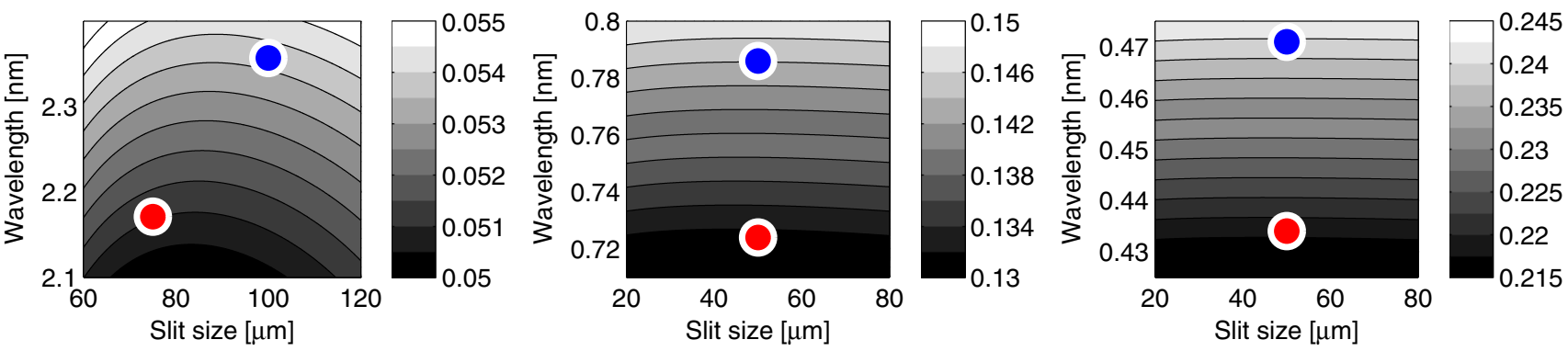

FIG. 7. Expected spectral resolution (color coded) as a function of wavelength and slit size as discussed in the setup section. The red and blue dots mark the chosen slit width for the warm and the cold undulator spectrum measurement, respectively. From left to right the best spectral resolution according to the wavelengths of the fundamental, third, and fifth harmonic is shown. The higher harmonics intrinsic undulator bandwidth of $1.67 \%$ and $1 \%$ for the third and the fifth harmonic, respectively is overlaid by the spectrometers resolution and thereby not resolvable. 
This approximation overestimates the diffraction broadening for the shorter wavelength of the higher harmonics, where a transition to the far field or rather Fraunhofer diffraction occurs, which is not considered in our approximation. The resolution of the spectrometer according to Eq. (A1) is plotted in Fig. 7. The blue and red dots (cooled and RT) mark the bandwidth of an ideal undulator detected by the TGS. A slit size of $75 \mu \mathrm{m}$ for the wavelength of the uncooled device's fundamental and $100 \mu \mathrm{m}$ for the cooled undulator's fundamental was chosen to optimize the spectrometers resolution whereas $50 \mu \mathrm{m}$ was chosen for the higher orders. An increase of the magnetic field error due to thermally induced mechanical distortions gives an additional summand in Eq. A1. This effect should be detectable if the field error (rms) becomes greater than $2 \%$. The bandwidths of the higher harmonics, intrinsically $1.67 \%$ and $1 \%$ for the third and the fifth harmonic, are dominated by the spectrometers resolution and could not be analyzed, see Fig. 7.

[1] A. R. Maier, A. Meseck, S. Reiche, C. B. Schroeder, T. Seggebrock, and F. Grüner, Phys. Rev. X 2, 031019 (2012).

[2] F. H. O'Shea, G. Marcus, J. B. Rosenzweig, M. Scheer, J. Bahrdt, R. Weingartner, A. Gaupp, and F. Grüner, Phys. Rev. ST Accel. Beams 13, 070702 (2010).

[3] F. Grüner, S. Becker, U. Schramm, T. Eichner, M. Fuchs, R. Weingartner, D. Habs, J. Meyer-ter Vehn, M. Geissler, M. Ferrario et al., Appl. Phys. B 86, 431 (2007).

[4] J. Bahrdt and Y. Ivanyushenkov, J. Phys. Conf. Ser. 425, 032001 (2013).

[5] K. Uestuener, M. Katter, R. Blank, D. Benedikt, J. Bahrdt, A. Gaupp, B. Klemke, F. Grüner, and R. Weingartner, Proceedings of the 20th Workshop on Rare Earth Permanent Magnets and Applications, Creta, Greece, 2008.

[6] J. Bahrdt, W. Frentrup, A. Gaupp, M. Scheer, R. Weingartner, F. O'Shea, and F. Grüner, in Proceedings of the 10th International Conference on Synchrotron Radiation and Instrumentation, Melbourne, Victoria, Australia, 2009.

[7] C. Abache and H. Oesterreicher, J. Appl. Phys. 57, 4112 (1985).

[8] F. O'Shea, G. Marcus, J. Rosenzweig, J. Bahrdt, A. Gaupp, F. Grüner, and R. Weingartner, in Proceedings of the 23rd Particle Accelerator Conference, Vancouver, Canada, 2009 (IEEE, Piscataway, NJ, 2009).

[9] R. Qiu, H.-S. Lee, T.-Y. Koo, and J. Li, AIP Conf. Proc. 879, 424 (2007).
[10] Y. Asano, T. Bizen, and X. Maréchal, J. Synchrotron Radiat. 16, 317 (2009).

[11] C. Kuhn, H.-J. Baecker, J. Bahrdt, A. Gaupp, and B. Schulz, Proceedings of the 4th International Particle Accelerator Conference, Shanghai, China, 2013, pp. 2126-2128.

[12] K.-J. Kim, AIP Conf. Proc. 184, 565 (1989).

[13] H. Onuki and P. Elleaume, Undulators, Wigglers and their Applications (Taylor and Francis, New York, 2003).

[14] J. A. Clarke, The Science and Technology of Undulators and Wigglers (Oxford University Press, New York, 2004).

[15] J. Bahrdt, in Proceedings of the 33rd International Free Electron Laser Conference, Shanghai, China, 2011, pp. 435-442.

[16] J. Bahrdt (private communication).

[17] H. Euteneuer, F. Hagenbuck, R. Herr, J. Herrmann, K. Herter, M. Ihm, K. Kaiser, C. Klümper, H. Krahn, H. Kreidel et al., in Proceedings of the Fourth European Particle Accelerator Conference EPAC 94, London, England (World Scientific, River Edge, NJ, 1994), Vol. 94, p. 506.

[18] Ultra-LEGe Detector, Canberra, 2002; http://www.canberra .com/products/detectors/pdf/Ultra-LEGe-SS-C39967.pdf.

[19] C. X. ray Center, The Chandra Proposers' Observatory Guide, http://cxc.harvard.edu/proposer/POG/html/chap9 .html.

[20] Manual: iKon BR-DD DW432, Andor (2008), back illuminated X-ray CCD.

[21] P. He, P. Cappadoro, T. Corwin, D. Harder, H. Fernandes, C. Kitegi, M. Kokole, J. Rank, G. Rakowsky, and T. Tanabe, Proceedings of the 4th International Particle Accelerator Conference, Shanghai, China, 2013, pp. 2304-2306.

[22] J. Revol, J. Biasci, J. Bouteille, F. Ewald, L. Farvacque, A. Franchi, G. Gautier, L. Goirand, M. Hahn, L. Hardy et al., Proceedings of the 4th International Particle Accelerator Conference, Shanghai, China, 2013, pp. 82-84.

[23] C. Benabderrahmane, M. Vallau, P. Berteaud, K. Tavakoli, J. L. Marlats, R. Nagaoka, N. Bchu, D. Zerbib, P. Brunelle, L. Chapuis et al., J. Phys. Conf. Ser. 425, 032019 (2013).

[24] M. Calvi, T. Schmidt, A. Anghel, A. Cervellino, S. J. Leake, P. R. Willmott, and T. Tanaka, J. Phys. Conf. Ser. 425, 032017 (2013).

[25] F. O'Shea, in Workshop on Physics and Applications of High Brightness Beams: Towards a Fifth Generation Light Source, Puerto Rico (2013).

[26] S. Sailaja, V. Arora, S. R. Kumbhare, P. A. Naik, P. D. Gupta, D. A. Fedin, A. A. Rupasov, and A. S. Shikanov, Meas. Sci. Technol. 9, 1462 (1998). 